International Journal of Bifurcation and Chaos, Vol. 12, No. 10 (2002) 2239-2253

(C) World Scientific Publishing Company

\title{
A GLOBAL SYNCHRONIZATION CRITERION FOR COUPLED CHAOTIC SYSTEMS VIA UNIDIRECTIONAL LINEAR ERROR FEEDBACK APPROACH
}

\author{
GUO-PING JIANG* and WALLACE K. S. TANG ${ }^{\dagger}$ \\ Department of Electronic Engineering, \\ City University of Hong Kong, \\ Kowloon, Hong Kong, China \\ *jianggp@njupt.edu.cn \\ ${ }^{\dagger} k s t a n g @ e e . c i t y u . e d u . h k$
}

Received August 17, 2001; Revised September 26, 2001

\begin{abstract}
Based on Lyapunov stabilization theory, this paper proposes a new generic criterion of global chaos synchronization between two coupled chaotic systems from a unidirectional linear error feedback coupling approach. The criterion is successfully applied to some typical chaotic systems with different types of nonlinearity, such as the classic Chua's circuit, the modified Chua's circuit with a sine function, and the Rössler and Lorenz chaotic systems. The coupling parameters are determined according to the new criterion so as to ensure the coupled systems' global chaos synchronization.
\end{abstract}

Keywords: Chaos; linear feedback; synchronization.

\section{Introduction}

This paper deals with the current popular problem of chaos synchronization, defined in the earlier papers [Pecora \& Carroll, 1990; Carroll \& Pecora, 1991]. Chaos synchronization can mainly be classified into two types called mutual synchronization and master-slave synchronization according to the coupling configuration [Ogorzalek, 1993; Wu \& Chua, 1993; Wu \& Chua, 1994; Chen, 1998; Chen \& Dong, 1998; Ushio, 1999]. The former is with bidirectional coupling [Chua et al., 1993], while the latter is with unidirectional coupling [Kapitaniak et al., 1996; Suykens \& Vandewalle, 1997; Grassi \& Mascolo 1997; Grassi \& Mascolo, 1999]. According to the linearity of the coupling signal, they can be classified into linear coupling and nonlinear coupling.
Due to the simple configuration and easy implementation, the unidirectional linear error feedback coupling scheme can be adopted in many real systems. In order to design a response or slave chaotic system by using the unidirectional linear error feedback methodology, the choice of the feedback gain or coupling parameters is a problem to be considered. Although some specific results have been given to determine the feedback gain or coupling parameters for particular systems such as Lur'e systems [Suykens \& Vandewalle, 1997; Curran et al., 1997; Suykens et al., 1998] a generic condition of global chaos synchronization has not been established for general chaotic systems. This strongly motivates the study on a new task of developing a generic criterion of global chaos synchronization for a general chaotic system, which, for a specific chaotic system, should easily deduce a specific feedback gain.

*On leave from Electronic Engineering Department, Nanjing University of Posts \& Telecommunications, Nanjing 210003, China 
In this paper, based on Lyapunov stability theory, we study the synchronization of two coupled chaotic systems using the unidirectional linear error feedback scheme. Our aim is to establish a generic condition of global chaos synchronization for a general chaotic system, and to apply the condition to some typical chaotic systems, for example, the classic Chua's circuit, the modified Chua's circuit with a sine function, Rössler and Lorenz chaotic systems such that synchronization is achieved.

The layout of this paper is as follows. In Sec. 2, based on Lyapunov stability theory, a generic condition of global chaos synchronization concerns two coupled systems using the unidirectional linear error feedback coupling scheme, and a novel global chaos synchronization criterion are established in the form of Lyapunov matrix inequality. Such conditions are applied to some typical chaotic systems with different types of nonlinear functions in Sec. 3, such as the classic Chua's circuit, the modified Chua's circuit with a sine function, the Rössler and Lorenz chaotic systems. To that end, conditions for choosing the feedback gain or coupling parameters are devised to ensure global chaos synchronization for these chaotic systems. Finally, conclusion remarks are then given in Sec. 4.

\section{Global Chaos Synchronization Criterion}

Considering a chaotic system with state equation in the form

$$
\dot{x}=A x+g(x)+u
$$

where $x \in R^{n}$ is the state vector, $u \in R^{n}$ is the external input vector, $A \in R^{n \times n}$ is a constant matrix and $g(x)$ is a continuous nonlinear function.

Assuming that

$$
g(x)-g(\tilde{x})=M_{x, \tilde{x}}(x-\tilde{x})
$$

for a bounded matrix $M_{x, \tilde{x}}$, in which the elements are dependent on $x$ and $\tilde{x}$. As illustrated in Sec. 3, most of the chaotic systems can be described by Eqs. (1) and (2).

Using the unidirectional linear error feedback coupling approach, a slave system for Eq. (1) can be constructed as follows:

$$
\dot{\tilde{x}}=A \tilde{x}+g(\tilde{x})+u+K(x-\tilde{x})
$$

where $K$ is a diagonal matrix with diagonal elements $d_{1}, d_{2}, \ldots, d_{n}$ being the coupling coefficients.
From Eqs. (1) and (3), the following error system equation is formed:

$$
\begin{aligned}
\dot{e} & =A e+g(x)-g(\tilde{x})-K e \\
& =A e+M_{x, \tilde{x}} e-K e \\
& =\left(A+M_{x, \tilde{x}}-K\right) e
\end{aligned}
$$

where $e=x-\tilde{x}$.

Theorem 1. If there exists a positive definite symmetric constant matrix $P$ such that

$$
\begin{aligned}
& \left(A-K+M_{x, \tilde{x}}\right)^{T} P \\
& \quad+P\left(A-K+M_{x, \tilde{x}}\right) \leq \mu I<0
\end{aligned}
$$

uniformly for all $x, \tilde{x}$ in the phase space, where $\mu$ denotes a negative constant, and $I$ is the identity matrix, then the error system (4) is globally exponentially stable.

Proof. Choose the Lyapunov function

$$
V=e^{T} P e
$$

where $P$ is a positive definite symmetric constant matrix.

Then, its derivative is

$$
\begin{aligned}
\dot{V}= & \dot{e}^{T} P e+e^{T} P \dot{e} \\
= & {\left[\left(A-K+M_{x, \tilde{x}) e]^{T} P e}\right.\right.} \\
& +e^{T} P\left[\left(A-K+M_{x, \tilde{x}}\right) e\right] \\
= & e^{T}\left[\left(A-K+M_{x, \tilde{x}}\right)^{T} P+P\left(A-K+M_{x, \tilde{x}}\right)\right] e \\
\leq & \mu\|e\|^{2} \\
< & 0
\end{aligned}
$$

where $\|\cdot\|$ denotes the Euclidean norm.

Based on the Lyapunov stability theory [Khalil, 1996; Martynyuk, 1998], it is known that, system (4) is globally exponentially stable, and hence, the two systems (1) and (3) are globally asymptotically synchronized.

\section{Synchronization of Typical Chaotic Systems}

To demonstrate the use of chaos synchronization criterion proposed herein, four examples of chaotic systems are considered. 


\subsection{Chua's circuit}

Chua's circuit [Shil'nikov, 1994] can be described by

$$
\left\{\begin{array}{l}
\dot{x}=\alpha(y-x-f(x)) \\
\dot{y}=x-y+z \\
\dot{z}=-\beta y
\end{array}\right.
$$

where $\alpha>0, \beta>0, a<b<0, f(\cdot)$ is a piecewise linear function described by

$$
f(x)=b x+\frac{1}{2}(a-b)(|x+1|-|x-1|) .
$$

In Eq. (9), we have

$$
f(x)-f(\tilde{x})=k_{x, \tilde{x}}(x-\tilde{x})
$$

where $k_{x, \tilde{x}}$ is dependant on $x$ and $\tilde{x}$, and varies in the interval $[a, b]$ for $t \geq 0$, that is, $k_{x, \tilde{x}}$ is bounded by the condition of $a \leq k_{x, \tilde{x}} \leq b<0$ (see Fig. 1).

Referring to Eq. (3), the following slave system is constructed for Eq. (8) with linear unidirectional coupling:

$$
\left\{\begin{array}{l}
\dot{\tilde{x}}=\alpha(\tilde{y}-\tilde{x}-f(\tilde{x}))+\delta_{x}(x-\tilde{x}) \\
\dot{\tilde{y}}=\tilde{x}-\tilde{y}+\tilde{z}+\delta_{y}(y-\tilde{y}) \\
\dot{\tilde{z}}=-\beta \tilde{y}+\delta_{z}(z-\tilde{z})
\end{array}\right.
$$

Subtracting Eq. (11) from Eq. (8), we obtain,

$$
\left\{\begin{array}{l}
\dot{e}_{x}=\alpha\left(e_{y}-e_{x}-k_{x, \tilde{x}} e_{x}\right)-\delta_{x} e_{x} \\
\dot{e}_{y}=e_{x}-e_{y}+e_{z}-\delta_{y} e_{y} \\
\dot{e}_{z}=-\beta e_{y}-\delta_{z} e_{z}
\end{array}\right.
$$

where $e_{x}=x-\tilde{x}, e_{y}=y-\tilde{y}$, and $e_{z}=z-\tilde{z}$.

Equation (12) can be rewritten as

$$
\dot{e}=A e+g(x)-g(\tilde{x})-K e
$$

where

$$
\begin{gathered}
A=\left[\begin{array}{rrr}
-\alpha & \alpha & 0 \\
1 & -1 & 1 \\
0 & -\beta & 0
\end{array}\right], \quad K=\left[\begin{array}{lll}
\delta_{x} & 0 & 0 \\
0 & \delta_{y} & 0 \\
0 & 0 & \delta_{z}
\end{array}\right], \\
e=\left[\begin{array}{l}
x-\tilde{x} \\
y-\tilde{y} \\
z-\tilde{z}
\end{array}\right]
\end{gathered}
$$

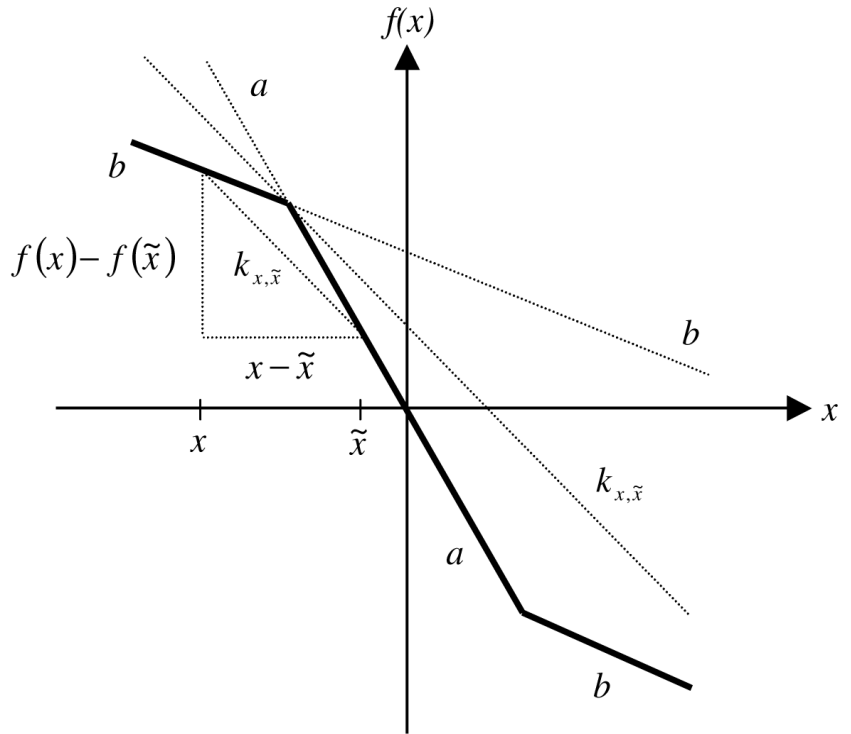

Fig. 1. Graphical representation of $k_{x, \tilde{x}}$ and Eq. (10).

and

$$
g(x)=\left[\begin{array}{c}
-\alpha f(x) \\
0 \\
0
\end{array}\right]
$$

Consider

$$
g(x)-g(\tilde{x})=\left[\begin{array}{c}
-\alpha(f(x)-f(\tilde{x})) \\
0 \\
0
\end{array}\right]
$$

$$
\begin{aligned}
& =\left[\begin{array}{c}
-\alpha k_{x, \tilde{x}}(x-\tilde{x}) \\
0 \\
0
\end{array}\right] \\
& =\left[\begin{array}{ccc}
-\alpha k_{x, \tilde{x}} & 0 & 0 \\
0 & 0 & 0 \\
0 & 0 & 0
\end{array}\right]\left[\begin{array}{l}
x-\tilde{x} \\
y-\tilde{y} \\
z-\tilde{z}
\end{array}\right] \\
& =M_{x, \tilde{x}} e
\end{aligned}
$$

where

$$
M_{x, \tilde{x}}=\left[\begin{array}{ccc}
-\alpha k_{x, \tilde{x}} & 0 & 0 \\
0 & 0 & 0 \\
0 & 0 & 0
\end{array}\right]
$$

From Eqs. (13) and (14), we get

$$
A-K+M_{x, \tilde{x}}=\left[\begin{array}{ccc}
-\alpha-\delta_{x}-\alpha k_{x, \tilde{x}} & \alpha & 0 \\
1 & -1-\delta_{y} & 1 \\
0 & -\beta & -\delta_{z}
\end{array}\right] .
$$


Choosing

$$
P=\left[\begin{array}{ccc}
p_{1} & 0 & 0 \\
0 & p_{2} & 0 \\
0 & 0 & p_{3}
\end{array}\right]
$$

where $p_{1}, p_{2}$ and $p_{3}$ are positive constants, then

$$
\begin{aligned}
(A- & \left.K+M_{x, \tilde{x}}\right)^{T} P+P\left(A-K+M_{x, \tilde{x}}\right)-\mu I \\
= & {\left[\begin{array}{ccc}
-\delta_{x}-\alpha k_{x, \tilde{x}}-\alpha & \alpha & 0 \\
1 & -1-\delta_{y} & 1 \\
0 & -\beta & -\delta_{z}
\end{array}\right]^{T}\left[\begin{array}{ccc}
p_{1} & 0 & 0 \\
0 & p_{2} & 0 \\
0 & 0 & p_{3}
\end{array}\right] } \\
& +\left[\begin{array}{ccc}
p_{1} & 0 & 0 \\
0 & p_{2} & 0 \\
0 & 0 & p_{3}
\end{array}\right]\left[\begin{array}{ccc}
-\delta_{x}-\alpha k_{x, \tilde{x}}-\alpha & \alpha & 0 \\
1 & -1-\delta_{y} & 1 \\
0 & -\beta & -\delta_{z}
\end{array}\right]-\left[\begin{array}{ccc}
\mu & 0 & 0 \\
0 & \mu & 0 \\
0 & 0 & \mu
\end{array}\right] \\
= & {\left[\begin{array}{ccc}
-2 p_{1}\left(\alpha k_{x, \tilde{x}}+\delta_{x}+\alpha+\frac{\mu}{2 p_{1}}\right) & p_{1} \alpha+p_{2} \\
p_{1} \alpha+p_{2} & -2 p_{2}\left(1+\delta_{y}+\frac{\mu}{2 p_{2}}\right) & p_{2}-p_{3} \beta \\
0 & p_{2}-p_{3} \beta
\end{array}\right] . }
\end{aligned}
$$

In Eq. (17), we have

$$
\begin{aligned}
& \Delta_{1}=-2 p_{1}\left(\alpha k_{x, \tilde{x}}+\delta_{x}+\alpha+\frac{\mu}{2 p_{1}}\right) \\
& \Delta_{2}=\operatorname{det}\left[\begin{array}{cc}
-2 p_{1}\left(\alpha k_{x, \tilde{x}}+\delta_{x}+\alpha+\frac{\mu}{2 p_{1}}\right) & p_{1} \alpha+p_{2} \\
p_{1} \alpha+p_{2} & -2 p_{2}\left(1+\delta_{y}+\frac{\mu}{2 p_{2}}\right)
\end{array}\right] \\
& =4 p_{1} p_{2}\left(\alpha k_{x, \tilde{x}}+\delta_{x}+\alpha+\frac{\mu}{2 p_{1}}\right)\left(1+\delta_{y}+\frac{\mu}{2 p_{2}}\right)-\left(p_{1} \alpha+p_{2}\right)^{2} \\
& \Delta_{3}=\operatorname{det}\left[\begin{array}{ccc}
-2 p_{1}\left(\alpha k_{x, \tilde{x}}+\delta_{x}+\alpha+\frac{\mu}{2 p_{1}}\right) & p_{1} \alpha+p_{2} & 0 \\
p_{1} \alpha+p_{2} & -2 p_{2}\left(1+\delta_{y}+\frac{\mu}{2 p_{2}}\right) & p_{2}-p_{3} \beta \\
0 & p_{2}-p_{3} \beta & -2 p_{3}\left(\delta_{z}+\frac{\mu}{2 p_{3}}\right)
\end{array}\right] \\
& =2 p_{1}\left(p_{2}-p_{3} \beta\right)^{2}\left(\delta_{x}+\alpha k_{x, \tilde{x}}+\alpha+\frac{\mu}{2 p_{1}}\right) \\
& -2 p_{3}\left(\delta_{z}+\frac{\mu}{2 p_{3}}\right)\left(4 p_{1} p_{2}\left(\delta_{x}+\alpha k_{x, \tilde{x}}+\alpha+\frac{\mu}{2 p_{1}}\right)\left(1+\delta_{y}+\frac{\mu}{2 p_{2}}\right)-\left(p_{1} \alpha+p_{2}\right)^{2}\right) .
\end{aligned}
$$


Assuming that $\Delta_{1}<0, \Delta_{2}>0$ and $\Delta_{3}<0$, we obtain

$$
\begin{aligned}
& \delta_{x}>-\alpha-\alpha k_{x, \tilde{x}}-\frac{\mu}{2 p_{1}} \\
& \delta_{y}>\frac{\left(p_{1} \alpha+p_{2}\right)^{2}}{4 p_{1} p_{2}\left(\delta_{x}+\alpha k_{x, \tilde{x}}+\alpha+\frac{\mu}{2 p_{1}}\right)}-1-\frac{\mu}{2 p_{2}} \\
& \delta_{z}>\frac{p_{1}\left(p_{2}-p_{3} \beta\right)^{2}\left(\delta_{x}+\alpha k_{x, \tilde{x}}+\alpha+\frac{\mu}{2 p_{1}}\right)}{p_{3}\left(4 p_{1} p_{2}\left(\delta_{x}+\alpha k_{x, \tilde{x}}+\alpha+\frac{\mu}{2 p_{1}}\right)\left(1+\delta_{y}+\frac{\mu}{2 p_{2}}\right)-\left(p_{1} \alpha+p_{2}\right)^{2}\right)}-\frac{\mu}{2 p_{3}} .
\end{aligned}
$$

Letting $p_{2}=\beta p_{3}$ for simplicity, Eq. (21) becomes

$$
\begin{aligned}
& \delta_{x}>-\alpha-\alpha k_{x, \tilde{x}}-\frac{\mu}{2 p_{1}} \\
& \delta_{y}>\frac{\left(p_{1} \alpha+p_{2}\right)^{2}}{4 p_{1} p_{2}\left(\delta_{x}+\alpha k_{x, \tilde{x}}+\alpha+\frac{\mu}{2 p_{1}}\right)}-1-\frac{\mu}{2 p_{2}} \\
& \delta_{z}>-\frac{\mu}{2 p_{3}} .
\end{aligned}
$$

Since $\alpha>0, \beta>0$ and $a \leq k_{x, \tilde{x}} \leq b<0$, we know that, if we choose suitable $\delta_{x}, \delta_{y}$ and $\delta_{z}$ such that

$$
\begin{aligned}
& \delta_{x}>-\alpha(a+1)-\frac{\mu}{2 p_{1}} \\
& \delta_{y}>\frac{\left(p_{1} \alpha+p_{2}\right)^{2}}{4 p_{1} p_{2}\left(\delta_{x}+a \alpha+\alpha+\frac{\mu}{2 p_{1}}\right)}-1-\frac{\mu}{2 p_{2}} \\
& \delta_{z}>-\frac{\mu}{2 p_{3}}
\end{aligned}
$$

then $\left(A-K+M_{x, \tilde{x}}\right)^{T} P+P\left(A-K+M_{x, \tilde{x}}\right)-\mu I<0$. According to Theorem 1, the two unidirectional coupled Chua's systems (8) and (11) are globally asymptotically synchronized.

Theorem 2. For two coupled Chua's systems (8) and (11), if $\delta_{x}, \delta_{y}$ and $\delta_{z}$ are chosen such that inequality (23) holds, then the two Chua's systems are globally asymptotically synchronized.

Assuming that $\alpha=9.78, \beta=14.97, a=-1.31$ and $b=-0.75$, the system (8) exhibits a chaotic behavior (see Fig. 2). By selecting $\mu=-0.2$, $P=\left[\begin{array}{ccc}2 & 0 & 0 \\ 0 & 14.97 & 0 \\ 0 & 0 & 1\end{array}\right]$ and the coupling parameters as $\delta_{x}=4.0, \delta_{y}=10.0$ and $\delta_{z}=1.0$, the inequality (23) holds. Based on Theorem 2, the two coupled Chua's circuits (8) and (11) with the above parameters are globally asymptotically synchronized, as shown in Fig. 3.
Considering the special case with $\delta_{y}=\delta_{z}=0$, the two $x$-coupled Chua's circuits become:

$$
\left\{\begin{array}{l}
\dot{x}=\alpha(y-x-f(x)) \\
\dot{y}=x-y+z \\
\dot{z}=-\beta y
\end{array}\right.
$$

and

$$
\left\{\begin{array}{l}
\dot{\tilde{x}}=\alpha(\tilde{y}-\tilde{x}-f(\tilde{x}))+\delta_{x}(x-\tilde{x}) \\
\dot{\tilde{y}}=\tilde{x}-\tilde{y}+\tilde{z} \\
\dot{\tilde{z}}=-\beta \tilde{y}
\end{array}\right.
$$

The error system of two $x$-coupled Chua's circuits (24) is

$$
\left\{\begin{array}{l}
\dot{e}_{x}=\alpha\left(e_{y}-e_{x}-k_{x, \tilde{x}} e_{x}\right)-\delta_{x} e_{x} \\
\dot{e}_{y}=e_{x}-e_{y}+e_{z} \\
\dot{e}_{z}=-\beta e_{y}
\end{array}\right.
$$

If $\delta_{x} \geq-\lambda a \alpha$ with $(\lambda>1)$, and $P=\left[\begin{array}{ccc}\beta & 0 & 0 \\ 0 & \alpha \beta & 0 \\ 0 & 0 & \alpha\end{array}\right]$, from Eqs. (7), (15) and (16), we get

$$
\begin{aligned}
\dot{V} & =-2\left(\alpha k_{x, \tilde{x}}+\delta_{x}\right) \beta e_{x}^{2}-2 \alpha \beta\left(e_{x}-e_{y}\right)^{2} \\
& \leq-2\left(a \alpha+\delta_{x}\right) \beta e_{x}^{2}-2 \alpha \beta\left(e_{x}-e_{y}\right)^{2} \\
& \leq-2(1-\lambda) a \alpha \beta e_{x}^{2}-2 \alpha \beta\left(e_{x}-e_{y}\right)^{2} \\
& =-2|(-a)(\lambda-1)| \alpha \beta e_{x}^{2}-2 \alpha \beta\left(e_{x}-e_{y}\right)^{2} \\
& \leq 0 .
\end{aligned}
$$

Thus we have $\dot{V}=0$ if $e_{x}=0, e_{y}=0$ and for any value of $e_{z}$. However, from Eq. (25), we have, if $e_{x} \equiv 0$ and $e_{y} \equiv 0$ then $e_{z} \equiv 0$. Therefore, by using LaSalle invariance principle [LaSalle, 1976], we know that the error system (25) is asymptotically stable, and hence the two $x$-coupled Chua's circuits (24) with the above parameters are also globally 

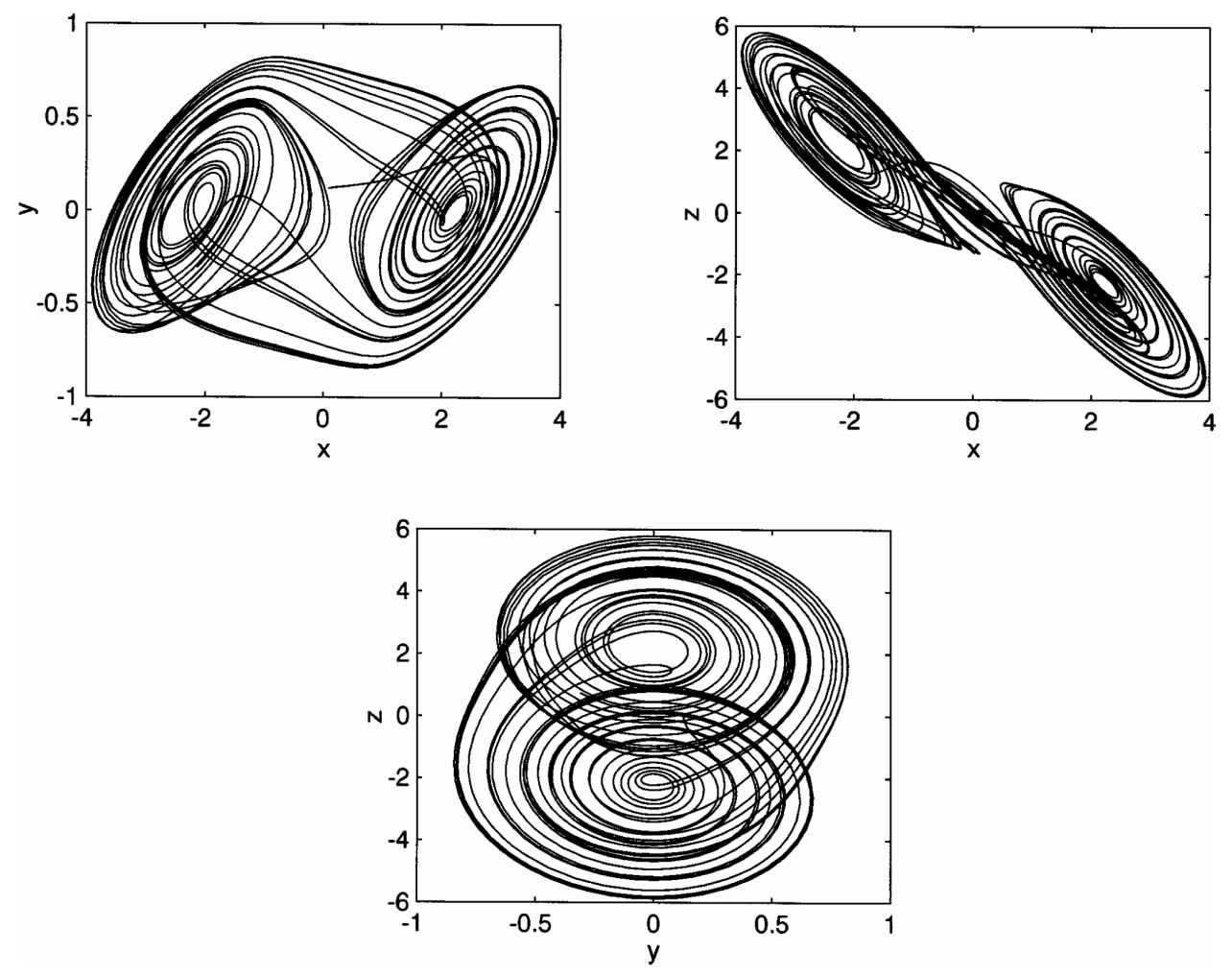

Fig. 2. The double scroll attractors of Chua's circuit.
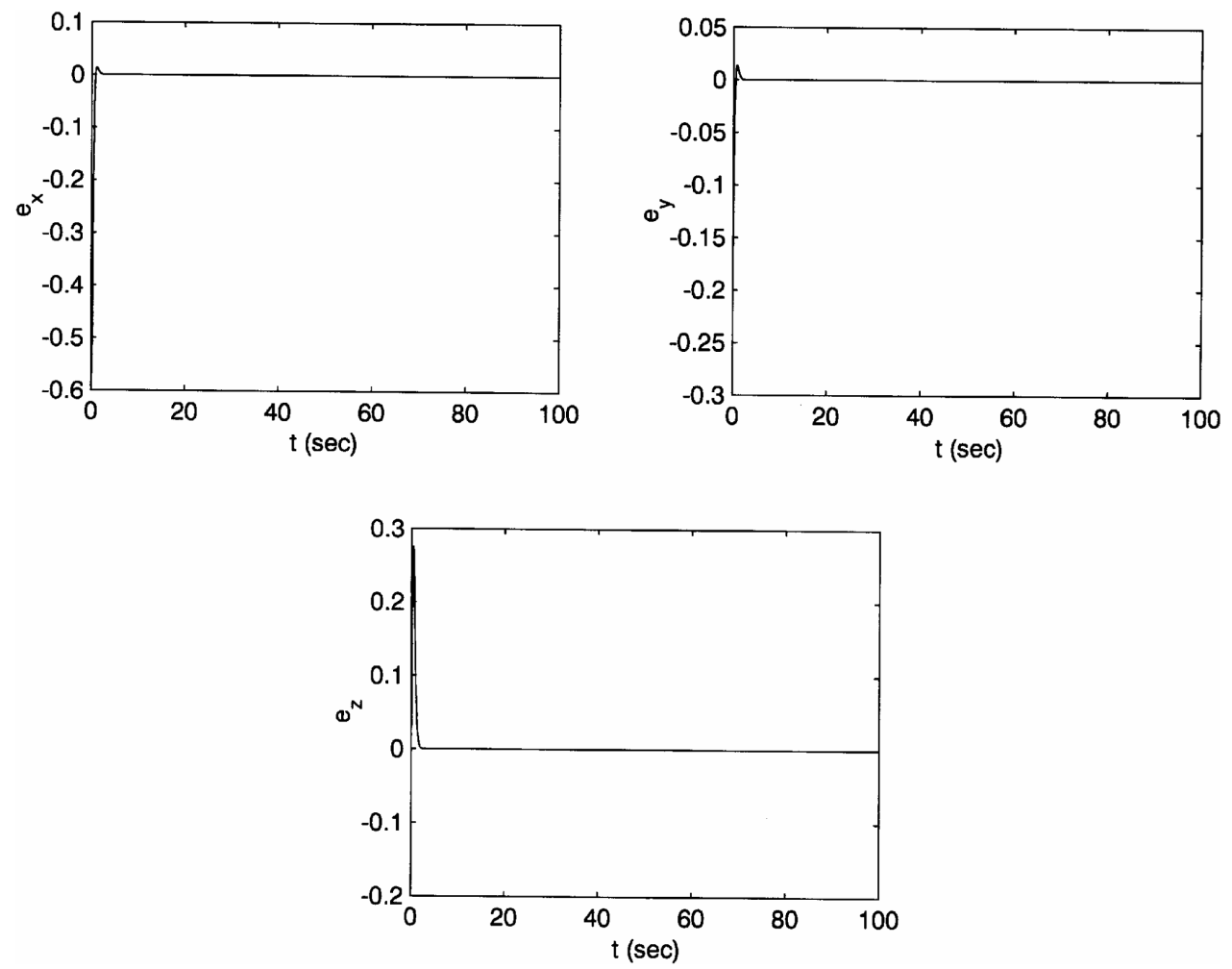

Fig. 3. The difference signals $e_{x}, e_{y}, e_{z}$ in two coupled Chua's circuits with the coupling coefficients $\delta_{x}=4.0, \delta_{y}=10.0$ and $\delta_{z}=1.0$. 

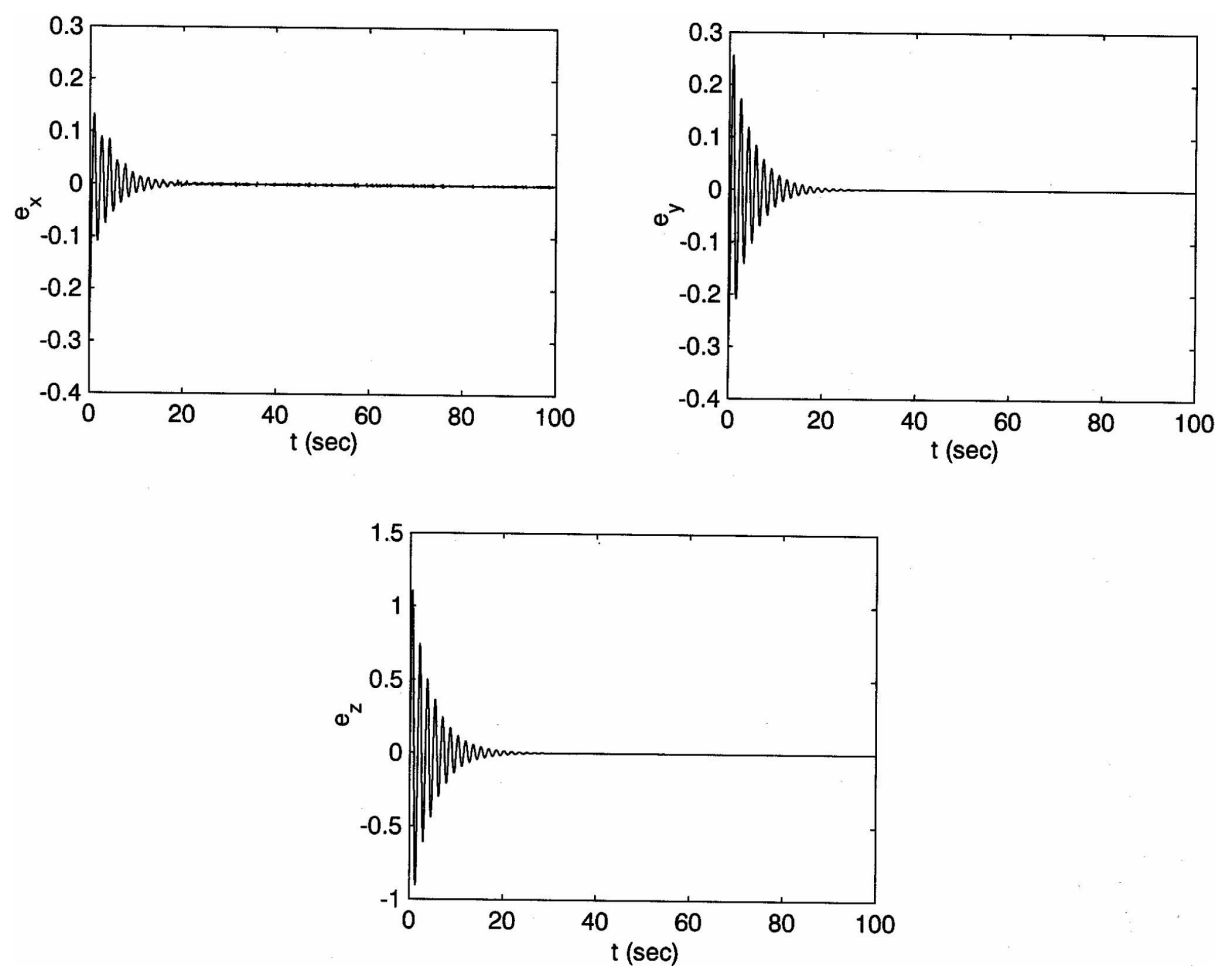

Fig. 4. The difference signals $e_{x}, e_{y}, e_{z}$ in two $x$-coupled Chua's circuits with the coupling coefficient $\delta_{x}=16.0$.

asymptotically synchronized. The simulation result with $\delta_{x}=16, \delta_{y}=0, \delta_{z}=0$ is shown in Fig. 4 .

\subsection{Modified Chua's circuit with a sine function}

Unlike the classic Chua's circuit, the nonlinear function of this modified Chua's circuit is governed by a trigonometric function [Tang et al., 2001b], which is a continuous function. It is reported that $n$-scroll attractors can be obtained, as shown in Fig. 5. The dimensionless state equation is given by

$$
\left\{\begin{array}{l}
\dot{x}=\alpha(y-f(x)) \\
\dot{y}=x-y+z \\
\dot{z}=-\beta y
\end{array}\right.
$$

where

$$
f(x)= \begin{cases}\frac{b \pi}{2 a}(x-2 a c) & \text { if } x \geq 2 a c \\ -b \sin \left(\frac{\pi x}{2 a}+d\right) & \text { if }-2 a c<x<2 a c \\ \frac{b \pi}{2 a}(x+2 a c) & \text { if } x \leq-2 a c .\end{cases}
$$

Here, in Eqs. (26) and (27), $\alpha, \beta, a, b, c, d$ are suitable constants, and $\alpha>0, \beta>0, a>0, b>0$.

An $n$-scroll attractor is generated with the following relationship:

$$
n=c+1
$$

and

$$
d= \begin{cases}\pi & \text { if } n \text { is odd } \\ 0 & \text { if } n \text { is even }\end{cases}
$$

In Eq. (27), we have

$$
f(x)-f(\tilde{x})=k_{x, \tilde{x}}(x-\tilde{x})
$$

where $k_{x, \tilde{x}}$ is dependent on $x$ and $\tilde{x}$, and satisfies the condition of $-\pi b / 2 a \leq k_{x, \tilde{x}} \leq \pi b / 2 a$ (similar to Fig. 1).

The slave system for system (26) by linear unidirectional coupling is

$$
\left\{\begin{array}{l}
\dot{\tilde{x}}=\alpha(\tilde{y}-f(\tilde{x}))+\delta_{x}(x-\tilde{x}) \\
\dot{\tilde{y}}=\tilde{x}-\tilde{y}+\tilde{z}+\delta_{y}(y-\tilde{y}) \\
\dot{\tilde{z}}=-\beta \tilde{y}+\delta_{z}(z-\tilde{z})
\end{array}\right.
$$



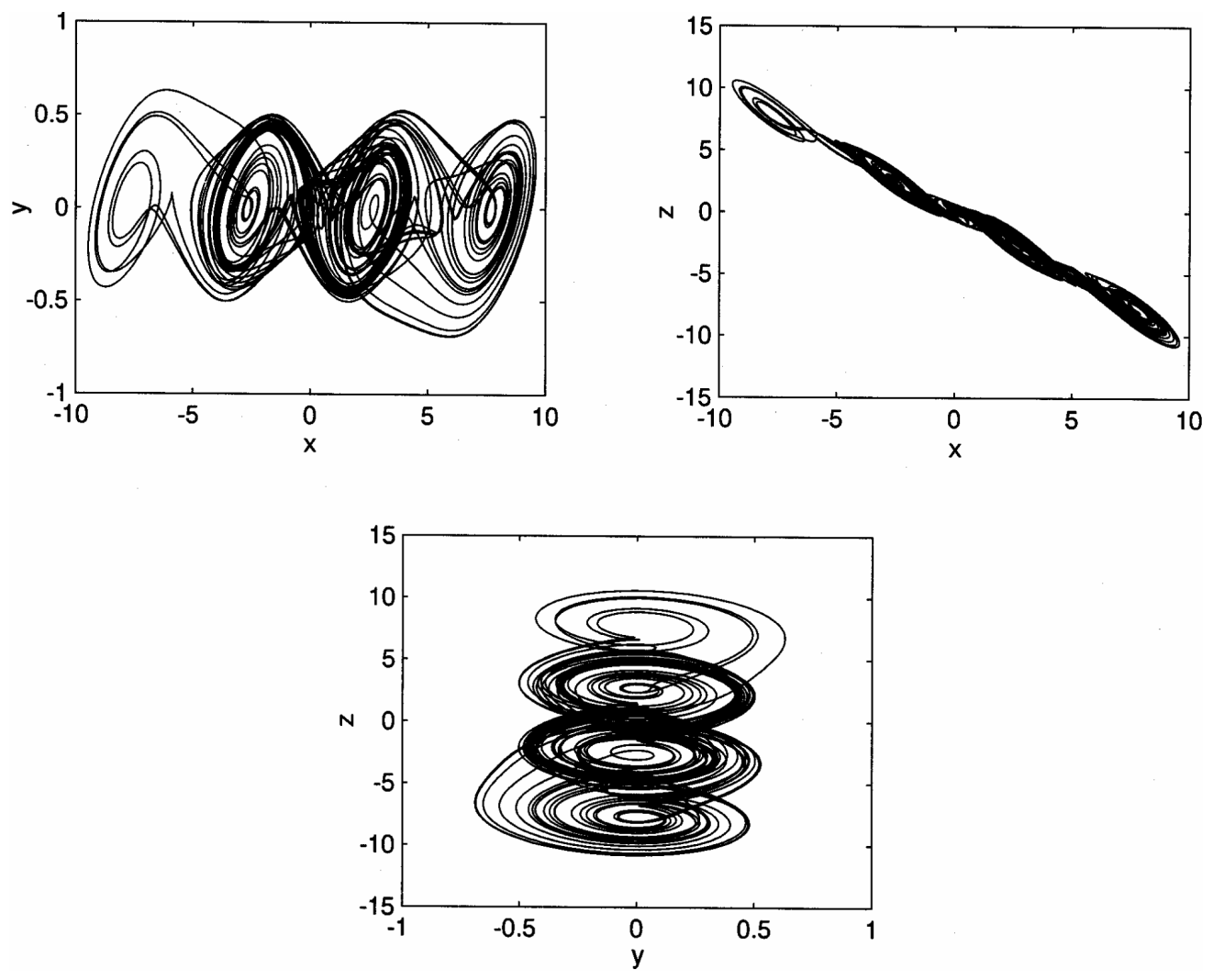

Fig. 5. Four-scroll attractors of the modified Chua's circuit with a sine function.

Subtracting Eq. (31) from Eq. (26), the following error system equation is obtained,

$$
\left\{\begin{array}{l}
\dot{e}_{x}=\alpha\left(e_{y}-k_{x, \tilde{x}} e_{x}\right)-\delta_{x} e_{x} \\
\dot{e}_{y}=e_{x}-e_{y}+e_{z}-\delta_{y} e_{y} \\
\dot{e}_{z}=-\beta e_{y}-\delta_{z} e_{z}
\end{array}\right.
$$

where $e_{x}=x-\tilde{x}, e_{y}=y-\tilde{y}$, and $e_{z}=z-\tilde{z}$.

Equation (32) can be rewritten as

$$
\dot{e}=A e+g(x)-g(\tilde{x})-K e
$$

where

$$
\begin{gathered}
A=\left[\begin{array}{ccc}
0 & \alpha & 0 \\
1 & -1 & 1 \\
0 & -\beta & 0
\end{array}\right], \quad K=\left[\begin{array}{ccc}
\delta_{x} & 0 & 0 \\
0 & \delta_{y} & 0 \\
0 & 0 & \delta_{z}
\end{array}\right], \\
e=\left[\begin{array}{l}
x-\tilde{x} \\
y-\tilde{y} \\
z-\tilde{z}
\end{array}\right]
\end{gathered}
$$

and

$$
g(x)=\left[\begin{array}{c}
-\alpha f(x) \\
0 \\
0
\end{array}\right]
$$

Hence, $g(x)-g(\tilde{x})=M_{x, \tilde{x}} e$ and

$$
M_{x, \tilde{x}}=\left[\begin{array}{ccc}
-\alpha k_{x, \tilde{x}} & 0 & 0 \\
0 & 0 & 0 \\
0 & 0 & 0
\end{array}\right] .
$$

From Eqs. (32) and (33), we get

$$
A-K+M_{x, \tilde{x}}=\left[\begin{array}{ccc}
-\delta_{x}-\alpha k_{x, \tilde{x}} & \alpha & 0 \\
1 & -1-\delta_{y} & 1 \\
0 & -\beta & -\delta_{z}
\end{array}\right] .
$$

Using a similar positive definite symmetric matrix $P$ as in Eq. (16) and following the same procedures, the below conditions are obtained 


$$
\begin{aligned}
& \delta_{x}>-\alpha k_{x, \tilde{x}}-\frac{\mu}{2 p_{1}} \\
& \delta_{y}>\frac{\left(p_{1} \alpha+p_{2}\right)^{2}}{4 p_{1} p_{2}\left(\delta_{x}+\alpha k_{x, \tilde{x}}+\frac{\mu}{2 p_{1}}\right)}-1-\frac{\mu}{2 p_{2}} \\
& \delta_{z}>\frac{p_{1}\left(p_{2}-p_{3} \beta\right)^{2}\left(\delta_{x}+\alpha k_{x, \tilde{x}}+\frac{\mu}{2 p_{1}}\right)}{p_{3}\left(4 p_{1} p_{2}\left(\delta_{x}+\alpha k_{x, \tilde{x}}+\frac{\mu}{2 p_{1}}\right)\left(1+\delta_{y}+\frac{\mu}{2 p_{2}}\right)-\left(p_{1} \alpha+p_{2}\right)^{2}\right)}-\frac{\mu}{2 p_{3}} .
\end{aligned}
$$

For simplicity, let $p_{2}=\beta p_{3}$, then from Eq. (35)

$$
\begin{aligned}
\delta_{x} & >-\alpha k_{x, \tilde{x}}-\frac{\mu}{2 p_{1}} \\
\delta_{y} & >\frac{\left(p_{1} \alpha+p_{2}\right)^{2}}{4 p_{1} p_{2}\left(\delta_{x}+\alpha k_{x, \tilde{x}}+\frac{\mu}{2 p_{1}}\right)}-1-\frac{\mu}{2 p_{2}} \\
\delta_{z} & >-\frac{\mu}{2 p_{3}} .
\end{aligned}
$$

Since $\alpha>0, \beta>0$ and $-\pi b / 2 a \leq k_{x, \tilde{x}} \leq \pi b / 2 a, \delta_{x}$, $\delta_{y}$ and $\delta_{z}$ are chosen such that

$$
\begin{aligned}
& \delta_{x}>\frac{\pi b \alpha}{2 a}-\frac{\mu}{2 p_{1}} \\
& \delta_{y}>\frac{\left(p_{1} \alpha+p_{2}\right)^{2}}{4 p_{1} p_{2}\left(\delta_{x}-\frac{\pi b \alpha}{2 a}+\frac{\mu}{2 p_{1}}\right)}-1-\frac{\mu}{2 p_{2}} \\
& \delta_{z}>-\frac{\mu}{2 p_{3}}
\end{aligned}
$$

then $\left(A-K+M_{x, \tilde{x}}\right)^{T} P+P\left(A-K+M_{x, \tilde{x}}\right)<\mu I$.

Theorem 3. For the two coupled modified Chua's systems (26) and (31), if $\delta_{x}, \delta_{y}$ and $\delta_{z}$ are chosen such that the inequality (37) holds, then the coupled modified Chua's systems are globally asymptotically synchronized.

Let $\alpha=10.814, \beta=14.0, a=1.3, b=0.11$, $c=3$ and $d=0$, the system (26) exhibits a chaotic

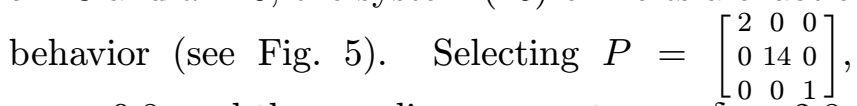
$\mu=-0.8$, and the coupling parameters as $\delta_{x}=2.8$, $\delta_{y}=9.0$ and $\delta_{z}=1.0$, then the inequality (37) holds. Hence using Theorem 3, the two coupled modified Chua's circuits (26) and (31) with the above parameters are globally asymptotically synchronized, as shown in Fig. 6.

Similar to the case in Chua's circuit, the two $x$-coupled modified Chua's circuits with the above parameters are also globally asymptotically synchronized with $\delta_{x}>\lambda \alpha(1+\pi b / 2 a)$ for $\lambda>1$, $\delta_{y}=\delta_{z}=0$ and $P=\left[\begin{array}{ccc}\beta & 0 & 0 \\ 0 & \alpha \beta & 0 \\ 0 & 0 & \alpha\end{array}\right]$. The simulation result, with $\delta_{x}=20$ and $\delta_{y}=\delta_{z}=0$, is shown in Fig. 7.

\subsection{Rössler system}

Rössler system [Rössler, 1976] is described by the following equation

$$
\left\{\begin{array}{l}
\dot{x}=-(y+z) \\
\dot{y}=x+\alpha y \\
\dot{z}=b+z(x-c)
\end{array}\right.
$$

where $a, b$ and $c$ denote positive parameters.

Using the unidirectional linear error feedback coupling approach, the slave system of (38) is constructed as follows:

$$
\left\{\begin{array}{l}
\dot{\tilde{x}}=-(\tilde{y}+\tilde{z})+\delta_{x}(x-\tilde{x}) \\
\dot{\tilde{y}}=\tilde{x}+a \tilde{y}+\delta_{y}(y-\tilde{y}) \\
\dot{\tilde{z}}=b+\tilde{z}(\tilde{x}-c)+\delta_{z}(z-\tilde{z}) .
\end{array}\right.
$$

In Eqs. (38) and (39), we assume

$$
\dot{e}=A e+g(x)-g(\tilde{x})-K e
$$

where

$$
\begin{gathered}
A=\left[\begin{array}{ccc}
0 & -1 & -1 \\
1 & a & 0 \\
0 & 0 & -c
\end{array}\right], \quad K=\left[\begin{array}{ccc}
\delta_{x} & 0 & 0 \\
0 & \delta_{y} & 0 \\
0 & 0 & \delta_{z}
\end{array}\right], \\
e=\left[\begin{array}{l}
x-\tilde{x} \\
y-\tilde{y} \\
z-\tilde{z}
\end{array}\right]
\end{gathered}
$$

and

$$
g(x)=\left[\begin{array}{c}
0 \\
0 \\
x z
\end{array}\right]
$$



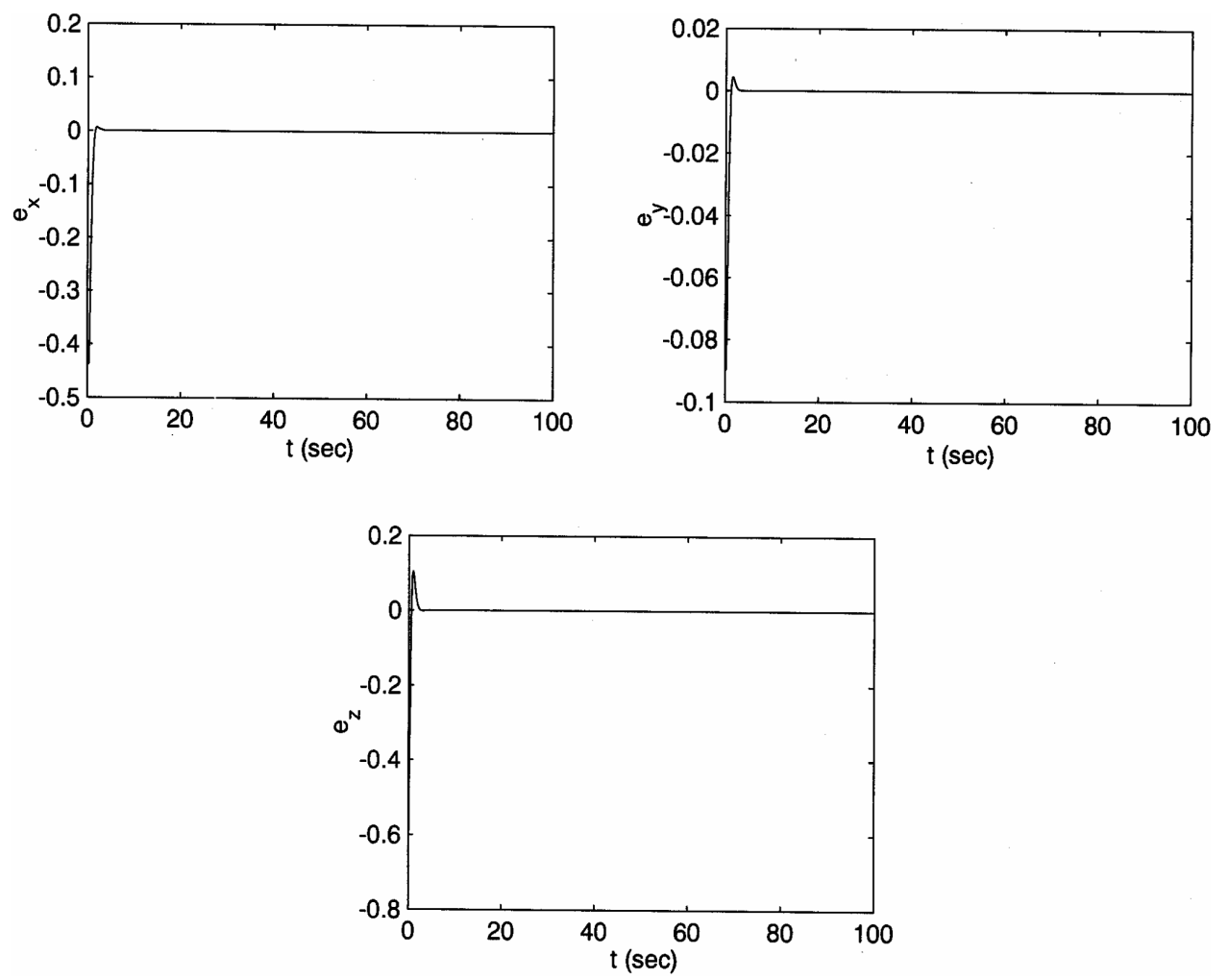

Fig. 6. The difference signals $e_{x}, e_{y}, e_{z}$ in two coupled modified Chua's circuits with the coupling coefficients $\delta_{x}=2.8$, $\delta_{y}=9.0$ and $\delta_{z}=1.0$.
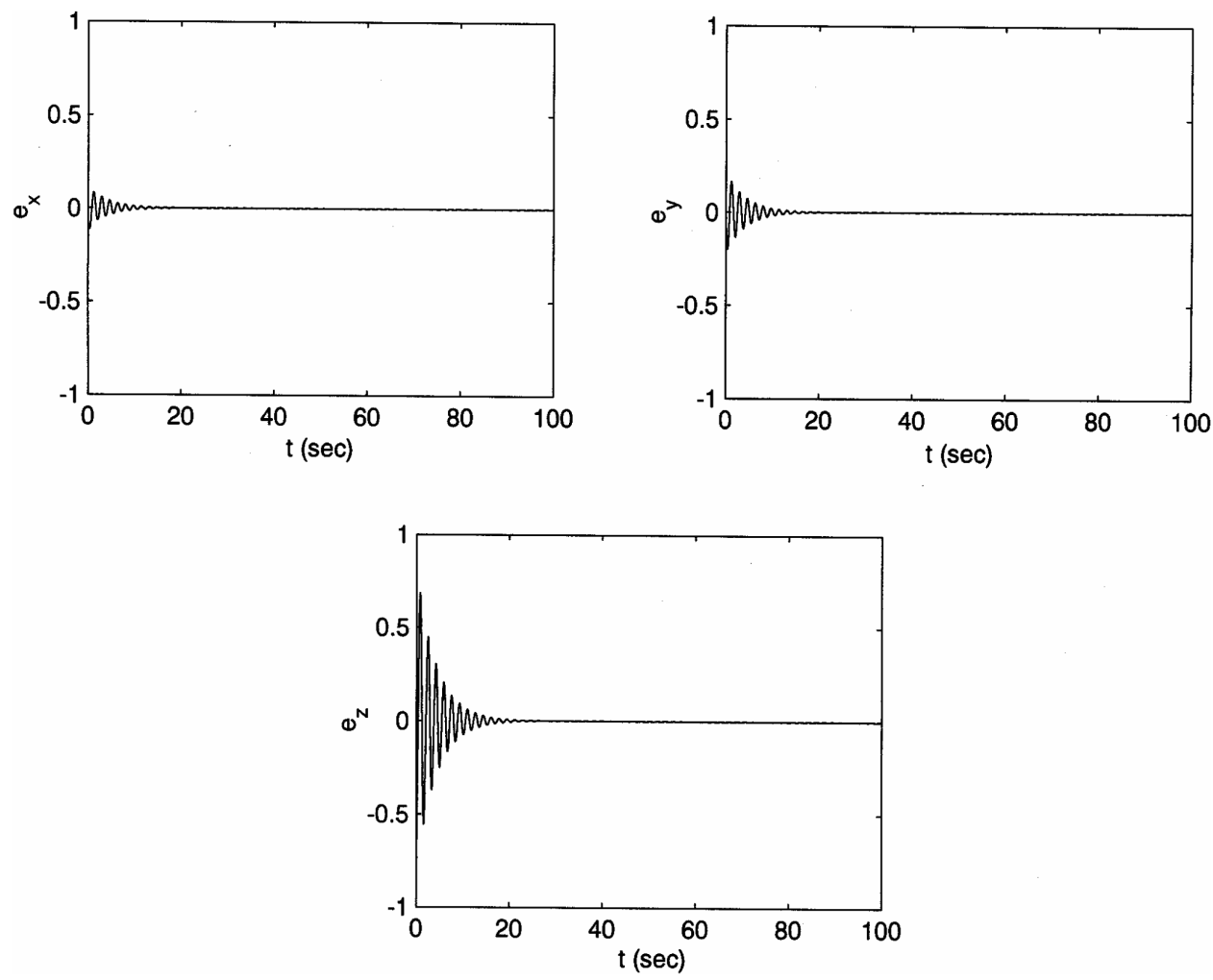

Fig. 7. The difference signals $e_{x}, e_{y}, e_{z}$ in two $x$-coupled modified Chua's circuits with the coupling coefficient $\delta_{x}=20$. 
Hence, $g(x)-g(\tilde{x})=M_{x, \tilde{x}} e$ and

$$
M_{x, \tilde{x}}=\left[\begin{array}{ccc}
0 & 0 & 0 \\
0 & 0 & 0 \\
\tilde{z} & 0 & x
\end{array}\right]
$$

We have

$$
A-K+M_{x, \tilde{x}}=\left[\begin{array}{ccc}
-\delta_{x} & -1 & -1 \\
1 & a-\delta_{y} & 0 \\
\tilde{z} & 0 & x-c-\delta_{z}
\end{array}\right]
$$

Using the same positive definite, symmetric matrix $P$ as in Eq. (16) and using the same procedures, the conditions of choosing suitable $\delta_{x}, \delta_{y}$ and $\delta_{z}$ such that $\left(A-K+M_{x, \tilde{x}}\right)^{T} P+P\left(A-K+M_{x, \tilde{x}}\right) \mu I$ are:

$$
\begin{aligned}
& \delta_{x}>-\frac{\mu}{2 p_{1}} \\
& \delta_{y}>a+\frac{\left(p_{2}-p_{1}\right)^{2}}{4 p_{1} p_{2}\left(\delta_{x}+\frac{\mu}{2 p_{1}}\right)}-\frac{\mu}{2 p_{2}} \\
& \delta_{z}>x-c+\frac{p_{2}\left(p_{3} \tilde{z}-p_{1}\right)^{2}\left(\delta_{y}+\frac{\mu}{2 p_{2}}-a\right)}{p_{3}\left(4 p_{1} p_{2}\left(\delta_{x}+\frac{\mu}{2 p_{1}}\right)\left(\delta_{y}-a+\frac{\mu}{2 p_{2}}\right)-\left(p_{2}-p_{1}\right)^{2}\right)}-\frac{\mu}{2 p_{3}} .
\end{aligned}
$$
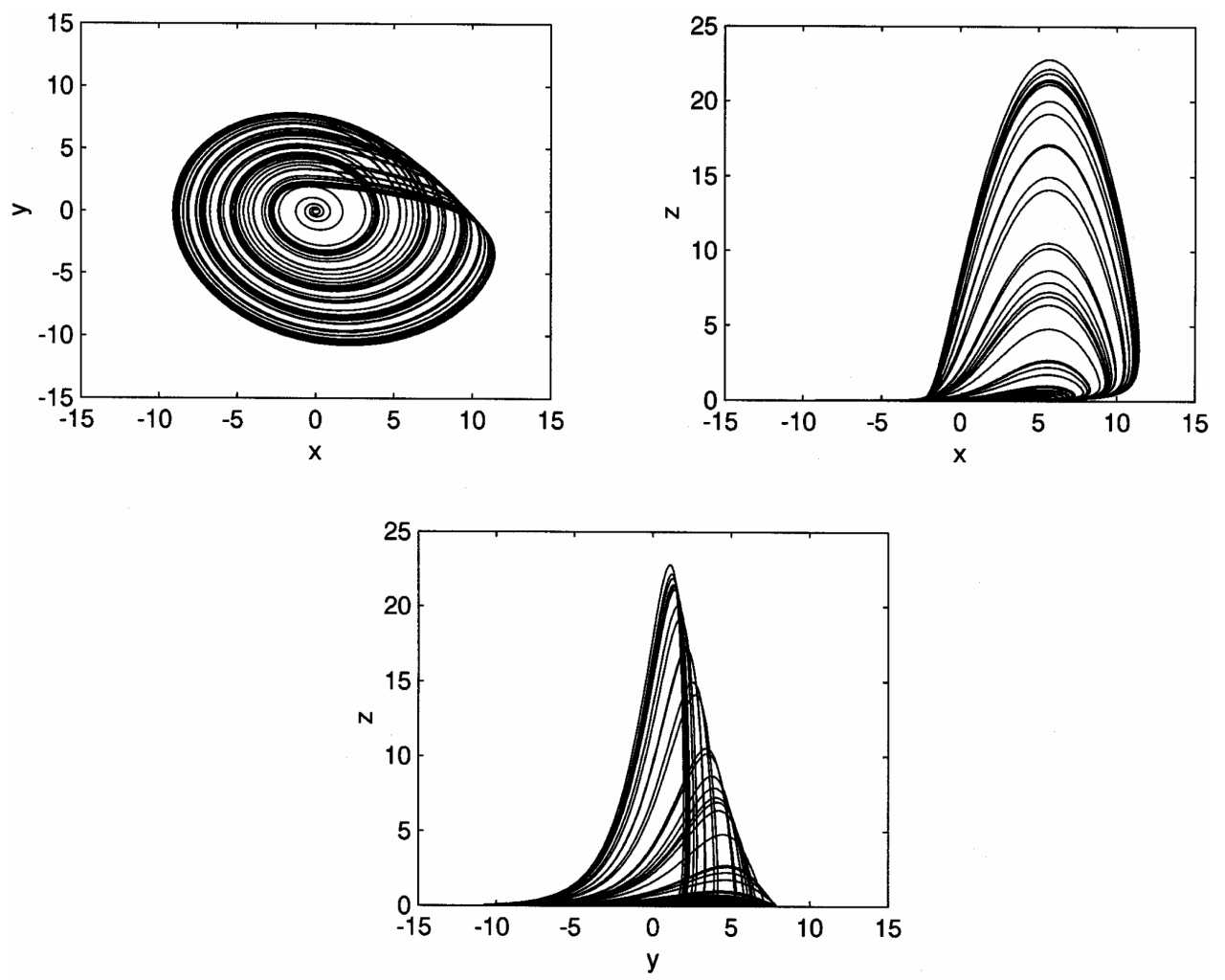

Fig. 8. The attractors of Rössler chaotic system. 

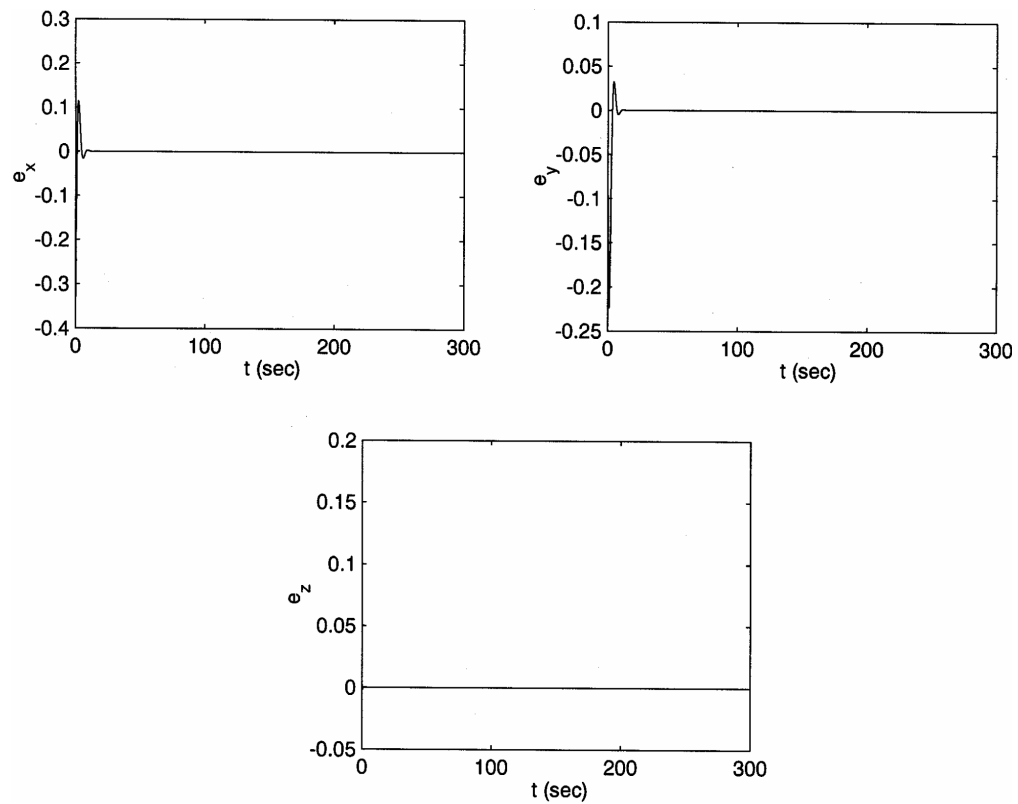

Fig. 9. The difference signals $e_{x}, e_{y}, e_{z}$ in two coupled Rössler chaotic systems with the coupling coefficients $\delta_{x}=1.0$, $\delta_{y}=0.3$ and $\delta_{z}=13$.

Since the motion trajectory of chaotic system is bounded, the third inequality in Eq. (42) holds for a large enough $\delta_{z}$.

Theorem 4. For the two coupled Rössler systems (38) and (39), if $\delta_{x}, \delta_{y}$ and $\delta_{z}$ are chosen such that the inequality (42) holds, then these two systems (38) and (39) are globally asymptotically synchronized.

Selecting $a=0.2, b=0.2, c=5.7$, the system exhibits a chaotic behavior as depicted in Fig. 8, from which, $-10<x<13,-12<y<8$, and $0<z<24$. Choosing $\mu=-0.2, p_{1}=p_{2}=2$, $p_{3}=0.1$, and the coupling parameters as $\delta_{x}=1.0$, $\delta_{y}=0.3, \delta_{z}=13$, obviously, the inequality (42) holds with these parameters, and hence, by using Theorem 4, we know that the two coupled Rössler systems (38) and (39) are globally asymptotically synchronized, as shown in Fig. 9.

\subsection{Lorenz system}

The Lorenz system [Lorenz, 1963; Cuomo et al., 1993] is given by

$$
\left\{\begin{array}{l}
\dot{x}=\sigma(y-x) \\
\dot{y}=\rho x-y-x z \\
\dot{z}=x y-\beta z
\end{array}\right.
$$

where $\sigma, \rho$ and $\beta$ are positive.

Using the unidirectional linear error feedback coupling approach, the slave system of Eq. (43) can be constructed as follows:

$$
\left\{\begin{array}{l}
\dot{\tilde{x}}=\sigma(\tilde{y}-\tilde{x})+\delta_{x}(x-\tilde{x}) \\
\dot{\tilde{y}}=\rho \tilde{x}-\tilde{y}-\tilde{x} \tilde{z}+\delta_{y}(y-\tilde{y}) \\
\dot{\tilde{z}}=\tilde{x} \tilde{y}-\beta \tilde{z}+\delta_{z}(z-\tilde{z}) .
\end{array}\right.
$$

In Eqs. (43) and (44), we assume

$$
\dot{e}=A e+g(x)-g(\tilde{x})-K e
$$

where

$$
\begin{gathered}
A=\left[\begin{array}{ccc}
-\sigma & \sigma & 0 \\
\rho & -1 & 0 \\
0 & 0 & -\beta
\end{array}\right], \quad K=\left[\begin{array}{ccc}
\delta_{x} & 0 & 0 \\
0 & \delta_{y} & 0 \\
0 & 0 & \delta_{z}
\end{array}\right], \\
e=\left[\begin{array}{l}
x-\tilde{x} \\
y-\tilde{y} \\
z-\tilde{z}
\end{array}\right]
\end{gathered}
$$

and

$$
g(x)=\left[\begin{array}{c}
0 \\
-x z \\
x y
\end{array}\right]
$$


Hence, $g(x)-g(\tilde{x})=M_{x, \tilde{x}} e$ and

$$
M_{x, \tilde{x}}=\left[\begin{array}{ccc}
0 & 0 & 0 \\
-z & 0 & -\tilde{x} \\
y & \tilde{x} & 0
\end{array}\right]
$$

We have

$$
A-K+M_{x, \tilde{x}}=\left[\begin{array}{ccc}
-\sigma-\delta_{x} & \sigma & 0 \\
\rho-z & -1-\delta_{y} & -\tilde{x} \\
y & \tilde{x} & -\beta-\delta_{z}
\end{array}\right]
$$

Using a similar positive definite, symmetric matrix $P$ as in Eq. (16) and letting $p_{2}=p_{3}$, the conditions of choosing suitable $\delta_{x}, \delta_{y}$ and $\delta_{z}$ such that $\left(A-K+M_{x, \tilde{x}}\right)^{T} P+P\left(A-K+M_{x, \tilde{x}}\right)<\mu I$ are:

$$
\begin{aligned}
& \delta_{x}>-\sigma-\frac{\mu}{2 p_{1}} \\
& \delta_{y}>\frac{\left(p_{1} \sigma+p_{2}(\rho-z)\right)^{2}}{4 p_{1} p_{2}\left(\delta_{x}+\sigma+\frac{\mu}{2 p_{1}}\right)}-1-\frac{\mu}{2 p_{2}} \\
& \delta_{z}>\frac{\left(1+\delta_{y}+\frac{\mu}{2 p_{2}}\right)\left(p_{2} y\right)^{2}}{4 p_{1} p_{2}\left(\sigma+\delta_{x}+\frac{\mu}{2 p_{1}}\right)\left(1+\delta_{y}+\frac{\mu}{2 p_{2}}\right)-\left(p_{1} \sigma+p_{2}(\rho-z)\right)^{2}}-\beta-\frac{\mu}{2 p_{3}} .
\end{aligned}
$$
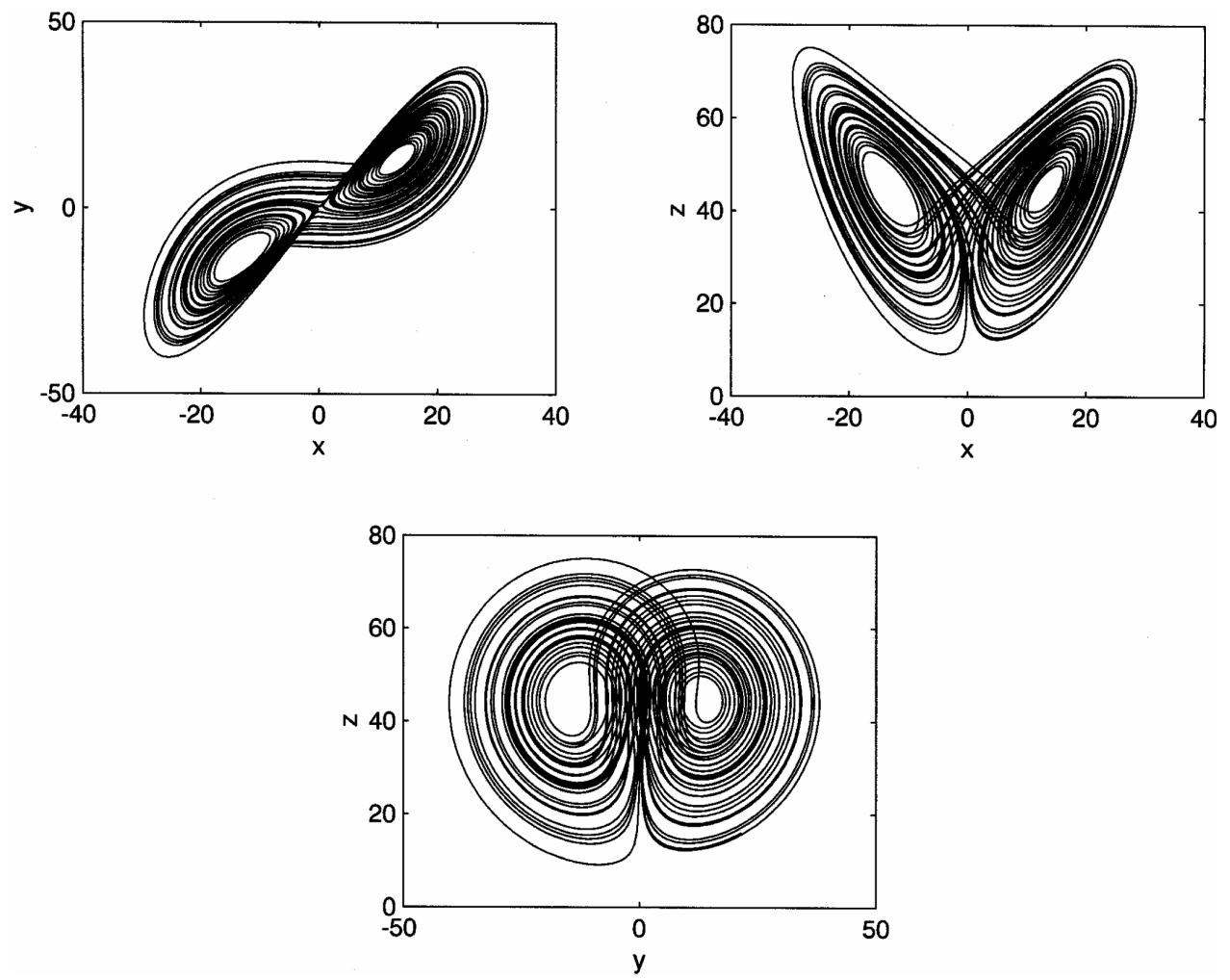

Fig. 10. The attractors of Lorenz chaotic system. 

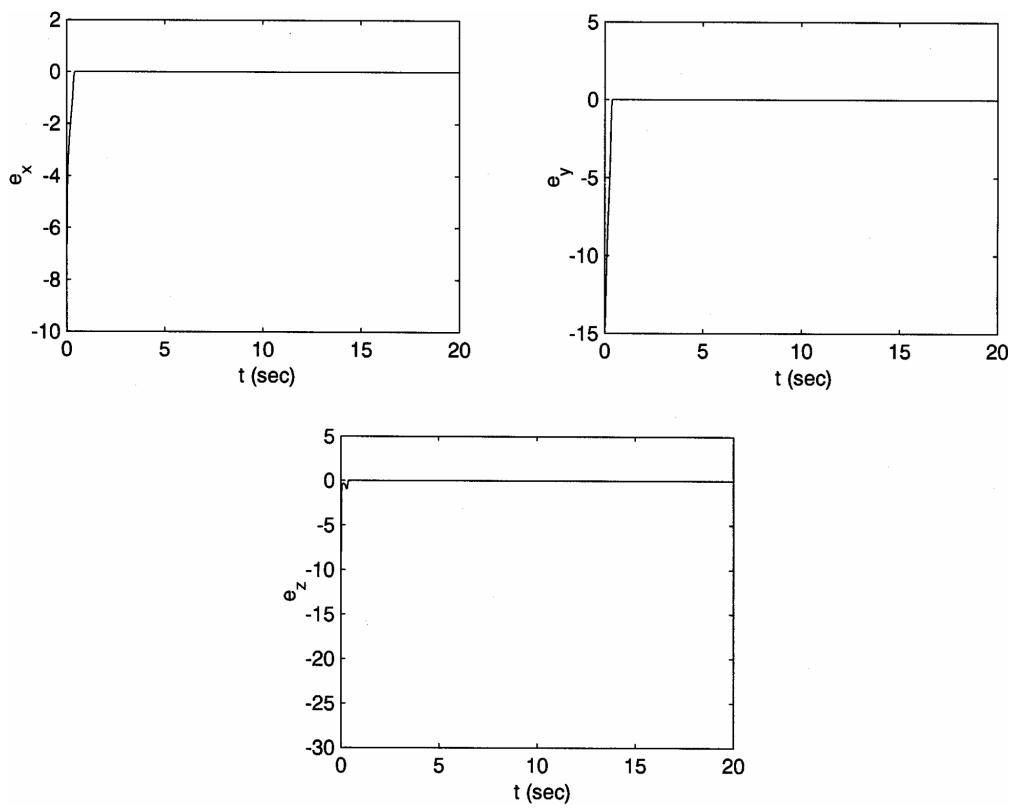

Fig. 11. The difference signals $e_{x}, e_{y}, e_{z}$ in two coupled Lorenz chaotic systems with the coupling coefficients $\delta_{x}=45.6$, $\delta_{y}=16.0$ and $\delta_{z}=110.0$.

Due to the fact that the motion trajectory of chaotic system is bounded, the second and third inequalities in Eq. (47) hold for sufficiently large $\delta_{y}$ and $\delta_{z}$.

Theorem 5. For the two coupled Lorenz systems (43) and (44), if $\delta_{x}, \delta_{y}$ and $\delta_{z}$ are chosen such that the inequality ( 47 ) holds, then these two systems (43) and (44) are globally asymptotically synchronized.

For $\sigma=16, \rho=45.6$ and $\beta=4$, the Lorenz system (43) exhibits a chaotic behavior, as shown in Fig. 10. From Fig. 10, $-30<x<35,-40<$ $y<50,0<z<90$. Hence, choosing $\mu=-0.1$, $p_{1}=p_{2}=p_{3}=1$, and the coupling parameters as $\delta_{x}=45.6, \delta_{y}=16.0$ and $\delta_{z}=110.0$, the inequality (47) holds. By using Theorem 5, the two coupled Lorenz systems (43) and (44) are globally asymptotically synchronized. The simulation results are given in Fig. 11.

\section{Conclusions}

In this paper, based on the unidirectional linear error feedback scheme for chaos synchronization, a new criterion is devised for choosing the suitable coupling parameters to ensure global chaos synchronization. It is also demonstrated that it can be applied to chaotic systems with different types of nonlinearity.
In general, the criterion proposed can be applied for all chaotic systems described by Eqs. (1) and (2). Hence, similar proof can be obtained for Murali-Lakshmanan-Chua (MLC) circuit [Murali et al., 1994; Murali \& Lakshmanan, 1997], the modified Chua's circuit with nonlinear quadratic function [Tang \& Man, 1998; Tang et al., 2001a], and so on. One can utilize the criterion to ensure the chaos synchronization, and easily obtain the methods for choosing feedback gain or coupling parameters for the synchronization of these coupled chaotic systems.

\section{Acknowledgments}

The authors are grateful to Prof. Guanrong Chen for the helpful discussions with him. This work is supported in part by University Key Teacher Foundation of Ministry of Education (MOE), P. R. China [Project No. NJUPT 2000-MOE-02] and a grant from the Research Grants Council of the Hong Kong Special Administrative Region, P. R. China [Project No. CityU 9040565].

\section{References}

Carroll, T. L. \& Pecora L. M. [1991] "Synchronization chaotic circuits," IEEE Trans. Circuits Syst. 38(4), 453-456.

Chen, G. [1998] Control and Synchronization of Chaotic 
Systems, A Bibliography, Department of Electrical Engineering, University of Houston, TX, USA, available via ftp:ftp.egr.uh.edu/pub/TeX/chaos.tex.

Chen, G. \& Dong, X. [1998] From Chaos to Order Methodologies, Perspectives and Applications (World Scientific, Singapore).

Chua, L. O., Itoh, M., Kocarev, L. \& Eckert, K. [1993] "Chaos synchronization in Chua's circuit," J. Circuits Syst. Comput. 3(1), 93-108.

Cuomo, K. M., Oppenheim, A. V. \& Strogatz, S. H. [1993] "Synchronization of Lorenz-based chaotic circuits with applications to communications," IEEE Trans. Circuits Syst. II 40(10), 626-633.

Curran, P. F., Suykens, J. A. K. \& Chua, L. O. [1997] "Absolute stability theory and master-slave synchronization," Int. J. Bifurcation and Chaos 7(12), 2891-2896.

Grassi, G. \& Mascolo, S. [1997] "Nonlinear observer design to synchronize hyperchaotic systems via a scalar signal," IEEE Trans. Circuits Syst. I 44(10), 1011-1014.

Grassi, G. \& Mascolo, S. [1999] "Synchronizing high dimensional chaotic systems via eigenvalue placement with application to cellular neural networks," Int. J. Bifurcation and Chaos 9(4), 705-711.

Kapitaniak, T., Sekieta, M. \& Ogorzalek, M. [1996] "Monotone synchronization of chaos," Int. J. Bifurcation and Chaos 6(1), 211-217.

Khalil, H. K. [1996] Nonlinear Systems, 2nd edition, (Prentice Hall, NJ).

LaSalle, J. P. [1976] The Stability of Dynamical Systems (SIAM, Philadelphia, PA).

Lorenz, E. N. [1963] "Deterministic nonperiodic flow," J. Atmos. Sci. 20, 130-141.

Martynyuk, A. A. [1998] Stability by Liapunov's Matrix Function Method with Applications (Marcel Dekker, NY).

Murali, K., Lakshmanan, M. \& Chua, L. O. [1994] "The simplest dissipative non-autonomous chaotic circuit," IEEE Trans. Circuits Syst. I 41(6), 462-463.

Murali, K. \& Lakshmanan, M. [1997] "Synchronization through compound chaotic signal in Chua's circuit and
Murali-Lakshmanan-Chua circuit," Int. J. Bifurcation and Chaos 7(2), 415-421.

Nijmeijer, H. \& Mareels, I. M. Y. [1997] "An observer looks at synchronization," IEEE Trans. Circuits Syst. I 44(10), 882-890.

Ogorzalek, M. J. [1993] "Taming chaos - Part I: Synchronization," IEEE Trans. Circuits Syst. I 40(10), 693-699.

Pecora, L. M. \& Carroll, T. L. [1990] "Synchronization in chaotic systems," Phys. Rev. Lett. 64(8), 821-824.

Rössler, O. E. [1976] "An equation for continuous chaos," Phys. Lett. A57, 397-398.

Shil'nikov, L. P. [1994] "Chua's circuit: Rigorous results and future problems," Int. J. Bifurcation and Chaos 4(3), 489-519.

Suykens, J. A. K. \& Vandewalle, J. [1997] "Master-slave synchronization of Lur'e systems," Int. J. Bifurcation and Chaos 7(3), 665-669.

Suykens, J. A. K., Yang, T. \& Chua, L. O. [1998] "Impulsive synchronization of chaotic Lur'e systems by measurement feedback," Int. J. Bifurcation and Chaos 8(6), 1371-1381.

Tang, K. S. \& Man, K. F. [1998] "An alternative Chua's circuit implementation," Proc. IEEE Int. Symp. Industrial Electronics (Pretoria, South Africa), pp. $441-444$.

Tang, K. S., Man, K. F., Zhong, G. Q. \& Chen, G. [2001a] "Generating chaos via $x|x|$," IEEE Trans. Circuits Syst. I 48(5), 636-641.

Tang, K. S., Zhong, G. Q., Chen, G. \& Man, K. F. [2001b] "Generation of $n$-scroll attractors via sine function," IEEE Trans. Circuits Syst. I 48(11), 1369-1372.

Ushio, T. [1999] "Synthesis of synchronized chaotic systems based on observers," Int. J. Bifurcation and Chaos 9(3), 541-546.

Wu, C. W. \& Chua, L. O. [1993] "A simple way to synchronize chaotic systems with applications to secure communication systems," Int. J. Bifurcation and Chaos 3(6), 1619-1627.

Wu, C. W. \& Chua, L. O. [1994] "A unified framework for synchronization and control of dynamical systems," Int. J. Bifurcation and Chaos 4(4), 979-998. 